\title{
Oxygen Radicals Inhibit Human Plasma Acetylhydrolase, the Enzyme That Catabolizes Platelet-activating Factor
}

\author{
Giuseppe Ambrosio, Alfonso Oriente, * Claudio Napoli, Giuseppe Palumbo, ${ }^{*}$ Paola Chiariello, Gianni Marone, * \\ Mario Condorelli, Massimo Chiariello, and Massimo Triggiani * \\ Department of Medicine, Divisions of Cardiology and *Clinical Immunology; and ${ }^{\ddagger}$ Department of Cellular and Molecular Pathology, \\ Federico II School of Medicine, 80131 Naples, Italy
}

\begin{abstract}
Platelet-activating factor (PAF) can exert profound inflammatory effects at very low concentrations. In plasma, PAF is hydrolyzed to lyso-PAF by acetylhydrolase, an enzyme that circulates bound to LDL. Previous studies suggest that oxygen radicals may act synergistically with PAF to potentiate tissue injury. However, mechanisms underlying this interaction have not been elucidated. In this study we investigated whether oxygen radicals may inactivate PAF acetylhydrolase. PAF acetylhydrolase activity was measured in human plasma and purified LDL before and after exposure to radicals $(10-20 \mathrm{nmol} / \mathrm{min}$ per $\mathrm{ml}$ ) generated by xanthine / xanthine oxidase. Oxygen radicals induced $>\mathbf{5 0} \%$ loss of PAF acetylhydrolase activity within $60 \mathrm{~s}$ and almost complete inactivation by $10 \mathrm{~min}$. This phenomenon was irreversible and independent of oxidative modification of LDL. Inactivation occurred without changes in the affinity constant of the enzyme ( $K_{\mathrm{m}}$ was $17.9 \mu \mathrm{M}$ under control conditions and 15.1 $\mu \mathrm{M}$ after exposure to oxygen radicals ). Inactivation was prevented by the scavengers superoxide dismutase or dimethylthiourea or by the iron chelator deferoxamine. Thus, superoxide-mediated, iron-catalyzed formation of hydroxyl radicals can rapidly and irreversibly inactivate PAF acetylhydrolase. Since concomitant production of PAF and oxygen radicals can occur in various forms of tissue injury, inactivation of acetylhydrolase might represent one mechanism by which oxygen radicals may potentiate and prolong the proinflammatory effects of PAF. (J. Clin. Invest. 1994. 93:2408-2416.) Key words: platelet-activating factor $\bullet$ oxygen radicals $\bullet$ acetylhydrolase $\bullet$ inflammation $\bullet$ neutrophils
\end{abstract}

\section{Introduction}

Platelet-activating factor (1-O-alkyl-2-acetyl-sn-glycero-3phosphocholine; $\mathrm{PAF})^{1}$ is a phospholipid synthesized by leu-

Address correspondence to Giuseppe Ambrosio, M.D., Ph.D., Cattedra di Cardiologia, Facoltà di Medicina Federico II, Via Sergio Pansini 5, 80131 Napoli, Italy.

Preliminary results from this work were presented at the 66th Meeting of the American Heart Association in Atlanta, GA on 8-11 November 1993.

Received for publication 19 April 1993 and in revised form 22 February 1994

1. Abbreviations used in this paper: DHF, dihydroxyfumaric acid; DMTU, dimethylthiourea; PAF, platelet-activating factor.

J. Clin. Invest.

(c) The American Society for Clinical Investigation, Inc.

0021-9738/94/06/2408/09 \$2.00

Volume 93, June 1994, 2408-2416 kocytes, endothelial cells, platelets, and other cell types. PAF was first described as a factor produced by activated basophils capable of inducing aggregation and degranulation of platelets (1-3), hence its name. Since then, several other important biological activities have been reported, including activation of neutrophils and monocytes $(4,5)$, marked vascular and bronchial effects (5-7), and depression of cardiac contractility (8, 9 ). As a consequence, PAF has been implicated in the pathophysiology of various diseases, such as asthma, endotoxic shock, and ischemia/reperfusion injury (5, 10-13).

The actions of PAF can be observed at very low concentrations, i.e., $10^{-10} \mathrm{M}$ or lower $(5,10,14)$. Therefore, the amount of PAF present in biological fluids needs to be tightly regulated. A major mechanism is through enzymatic conversion to inactive compounds. Human plasma contains an enzyme, PAF acetylhydrolase (1-alkyl-2-acetyl-sn-glycero-3-phosphocholine acetohydrolase, EC 3.1.1.47), that specifically removes the acetyl group at the $s n-2$ position of PAF, converting it to lysoPAF (15-18). Plasma PAF acetylhydrolase is a 43,000-mol wt protein that circulates bound to lipoproteins because of its highly hydrophobic nature. The physiologically relevant PAF acetylhydrolase activity in plasma is associated with LDL (17). LDL-associated PAF acetylhydrolase activity, therefore, ensures that plasma concentrations of PAF are kept low under normal circumstances and that PAF produced by activated cells is rapidly hydrolyzed.

In addition to PAF production, tissue injury and inflammation are accompanied by the synthesis and release of other chemical mediators. These species are not only responsible for a specific, mediator-dependent component of tissue injury, but they may also cooperate with each other in amplifying the inflammatory response. Increasing evidence suggests that a major role in various types of tissue injury is played by oxygen radicals. Large amounts of oxygen radicals are known to be generated upon reperfusion of ischemic organs $(19,20)$ or during inflammatory reactions by leukocytes and resident cells that have become activated $(21,22)$. Several studies have suggested that oxygen radicals and PAF may cooperatively mediate vascular injury in intestinal ischemia and reperfusion (23, 24) and bronchial hyperresponsiveness in asthma (25-27), and it has been proposed that a similar phenomenon may also be involved in other types of inflammatory injury. However, possible mechanisms of the interaction between PAF and oxygen radicals have not been investigated.

Oxygen free radicals are very reactive chemical species. Once formed, they may rapidly interact with other molecules to induce a number of alterations, including modifications of proteins and enzyme inactivation (28-31). If acetylhydrolase were also susceptible to inactivation by oxygen radicals, this phenomenon might reduce PAF catabolism and therefore prolong and/or potentiate the inflammatory effects of this media- 
tor. Therefore, the aim of this study was to investigate whether exposure to oxygen radicals would affect LDL-associated PAF acetylhydrolase activity.

\section{Methods}

LDL isolation. Blood was obtained from normal volunteers after an overnight fasting. LDL were isolated from serum by density ultracentrifugations in $\mathrm{KBr}$ (32), followed by overnight dialysis at $4^{\circ} \mathrm{C}$ against buffer containing $50 \mathrm{mM}$ potassium phosphate, $150 \mathrm{mM} \mathrm{NaCl}$, and 1 mM EDTA, pH 7.4. LDL were stored under nitrogen at $4^{\circ} \mathrm{C}$ until use (within $2 \mathrm{wk}$ ). Before assay, LDL were concentrated to $\sim 1.8 \mathrm{mg} / \mathrm{ml}$ by centrifugation in Centricon ${ }^{\otimes}$ tubes and checked to confirm purity and to rule out spontaneous autoxidation. LDL always consisted of a single band when analyzed by acrylamide gel electrophoresis and by agarose gel electrophoresis $(33,34)$. Presence of lipid peroxidation products was determined as described previously (35) and found to be always $<2.0 \mathrm{nmol}$ of thiobarbiturate-reactive material $/ \mathrm{mg}$ of protein. Protein content was measured by the Lowry assay (36), using BSA as a standard.

Oxygen radical generation. In most experiments, oxygen radicals were generated in the reaction of xanthine with xanthine oxidase. $30 \mu \mathrm{g}$ of LDL (in $1 \mathrm{ml}$ ) was used in all experiments. Xanthine was added at a final concentration of $200 \mu \mathrm{M}$, unless specified otherwise. The reaction was started by addition of appropriate amounts (typically, $100 \mathrm{mU} /$ $\mathrm{ml}$ ) of xanthine oxidase (salicylate-free, from bovine milk; sp act 1 $\mathrm{U} / \mathrm{mg}$ protein) (Boehringer Mannheim $\mathrm{GmbH}$, Mannheim, Germany) and was allowed to proceed at $37^{\circ} \mathrm{C}$ for variable amounts of time. The xanthine/xanthine oxidase reaction yields both superoxide radicals and hydrogen peroxide $(37,38)$, which in turn may give rise to hydroxyl radicals in the presence of trace amounts of iron or other transition metals (39). Therefore, the relative role of each oxidant species on PAF acetylhydrolase activity was dissociated by performing parallel experiments in which LDL were incubated with xanthine/ xanthine oxidase in the presence of one of the following agents: the superoxide radical scavenger SOD ( $\mathrm{Cu}-\mathrm{Zn}$ form, from bovine erythrocytes; sp act $3,570 \mathrm{U} / \mathrm{mg}$ of protein); the hydrogen peroxide scavenger catalase (from bovine liver, sp act $40,000 \mathrm{U} / \mathrm{mg}$ of protein); the hydroxyl radical scavenger dimethylthiourea (DMTU, $10 \mathrm{mM}$ ); or the iron chelator deferoxamine $(100 \mu \mathrm{M})$. Superoxide radical production by the xanthine/xanthine oxidase system was measured by the rate of SOD-inhibitable reduction of cytochrome $c(1.2 \mathrm{mM})$ at $550 \mathrm{~nm}$, at $37^{\circ} \mathrm{C}$, in a double-beam spectrophotometer equipped with a stripchart recorder (UVIKON 810; Kontron Instruments, Zürich, Switzerland) (37).

Acetylhydrolase assay. Acetylhydrolase activity was assayed as described previously (40), with slight modifications. Samples were incubated with [ $\left.{ }^{3} \mathrm{H}\right] \mathrm{PAF}$ ( $5 \mathrm{nmol} / 0.5 \mathrm{ml}$, unless otherwise indicated $)$ complexed with essentially fatty acid-free $\mathrm{HSA}(0.1 \mathrm{mg} / \mathrm{ml})$ for $15 \mathrm{~min}$ at $37^{\circ} \mathrm{C}$. The reaction was stopped by addition of $3 \mathrm{vol}$ of methanol/ chloroform $(2: 1, \mathrm{vol} / \mathrm{vol})$. The lipid fraction was extracted by a modification of the method of Bligh and Dyer (41), in which sufficient formic acid was added to lower the $\mathrm{pH}$ of the aqueous layer to 3. Unlabeled PAF ( $10 \mu \mathrm{g} /$ tube) was added immediately before the lipid extraction to increase the recovery of radioactivity. Solvents were removed by a stream of nitrogen, and the lipids were resuspended in chloroform/ methanol (1:1, vol/vol). Recovery of added radioactivity always exceeded $85 \%$. The radioactive compounds recovered from the extraction were then separated by TLC on layers of Silica Gel G developed in chloroform/methanol/acetic acid/water (50:25:8:3; vol/vol), as described previously (42). Standards of PAF and lyso-PAF were visualized with iodine vapors, and the distribution of label in different products was determined by scanning the plate for radioactivity using an imaging scanner (System 2000; Bioscan, Washington, DC). Labeled products were then isolated and scraped from the TLC plate, and the amount of $\left[{ }^{3} \mathrm{H}\right]$ lyso-PAF formed during the reaction was determined by liquid scintillation counting.
Chemicals. Radiolabeled PAF (1- $\left[{ }^{3} \mathrm{H}\right]$-alkyl-2-acetyl-sn-glycero-3phosphocholine; $60 \mathrm{Ci} / \mathrm{mmol}$ ) was purchased from New England $\mathrm{Nu}$ clear (Boston, MA). Unlabeled PAF, lyso-PAF, essentially fatty acidfree HSA, and the various anti-free radical agents were purchased from Sigma Chemical Co. (St. Louis, MO). All solvents were of HPLC grade from Carlo Erba (Milan, Italy).

Statistical analysis. Data are expressed as mean \pm SEM. Differences among various groups were tested by one-way ANOVA, followed by Student's $t$ test to determine differences between groups. Values of $K_{\mathrm{m}}$ of acetylhydrolase activity were estimated by linear regression analysis of Lineweaver-Burk plots.

\section{Results}

Effects of oxygen radicals on PAF acetylhydrolase activity. Plasma PAF acetylhydrolase in normal volunteers converted $0.48 \pm 0.07 \mathrm{pmol}$ of $\left[{ }^{3} \mathrm{H}\right] \mathrm{PAF}$ to $\left[{ }^{3} \mathrm{H}\right]$ lyso-PAF $/ \mathrm{min}$ per $\mu \mathrm{g}$ of protein. PAF acetylhydrolase activity was enriched $\sim 40$-fold in purified LDL, to $18.8 \pm 1.3$ pmol of $\left[{ }^{3} \mathrm{H}\right] \mathrm{PAF}$ converted to $\left[{ }^{3} \mathrm{H}\right.$ ]lyso-PAF $/ \mathrm{min}$ per $\mu \mathrm{g}$ of protein. We then tested the effects of oxygen radicals on enzyme activity. Under our experimental conditions, $30 \mu \mathrm{g}$ of control LDL converted $68.2 \pm 5.3 \%$ of $\left[{ }^{3} \mathrm{H}\right]$ PAF to $\left[{ }^{3} \mathrm{H}\right]$ lyso-PAF over $15 \mathrm{~min}$. In contrast, when PAF was incubated with an identical amount of LDL that had been exposed to the xanthine/xanthine oxidase system, PAF acetylhydrolase activity was virtually abolished (Fig. 1).

To test whether xanthine oxidase-induced inactivation was specifically due to oxygen radicals, PAF acetylhydrolase activity was also determined on LDL that had been exposed to the oxygen radical-generating system in the presence of the superoxide radical scavenger SOD and/or the hydrogen peroxide scavenger catalase. In the absence of xanthine oxidase, neither SOD nor catalase affected PAF acetylhydrolase activity (data not shown). Addition of both SOD and catalase completely prevented xanthine oxidase-induced inactivation of PAF acetylhydrolase (Fig. 1). Similar results were obtained when SOD alone was present, whereas catalase alone was without effect (Fig. 1). Inactivation could be reproduced by exposing PAF acetylhydrolase for $3 \mathrm{~h}$ to $5 \mathrm{mM}$ dihydroxyumaric acid (DHF), another generator of oxygen radicals $(43,44)$. Conversion of

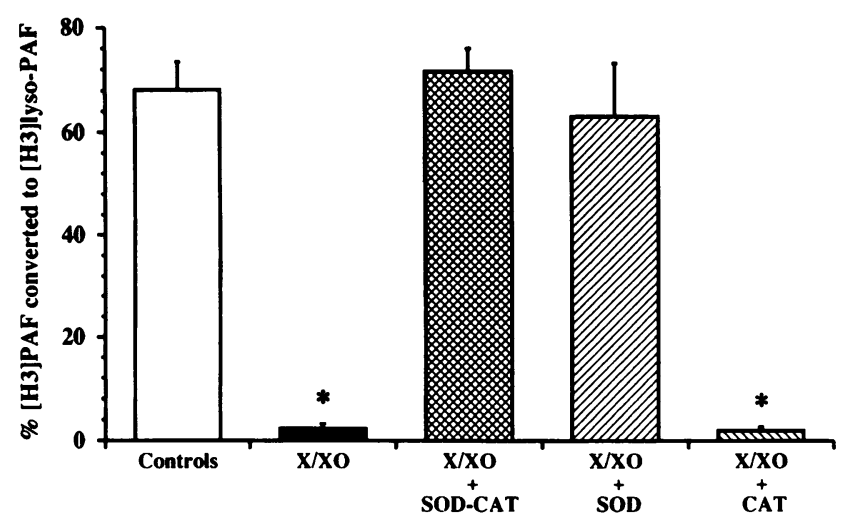

Figure 1. Effects of exposure to oxygen radicals on LDL-associated PAF acetylhydrolase activity. Conversion of $\left[{ }^{3} \mathrm{H}\right] \mathrm{PAF}$ to $\left[{ }^{3} \mathrm{H}\right]$ lysoPAF was virtually abolished by prior incubation with xanthine $(X$; $200 \mu \mathrm{M})$ plus xanthine oxidase $(X O ; 100 \mathrm{mU} / \mathrm{ml})$ for $18 \mathrm{~h}$ at $37^{\circ} \mathrm{C}$. In parallel experiments, oxygen radicals were inactivated by the specific scavenger enzymes $\mathrm{SOD}(330 \mathrm{U} / \mathrm{ml})$ and catalase $(C A T ; 1,000$ $\mathrm{U} / \mathrm{ml})$. Data are the mean $\pm \mathrm{SE}$ of six experiments. *Significantly different from controls; $P<0.05$. 
$\left[{ }^{3} \mathrm{H}\right] \mathrm{PAF}$ to $\left[{ }^{3} \mathrm{H}\right]$ lyso-PAF over $15 \mathrm{~min}$ was reduced from $55.0 \pm 2.6 \%$ under control conditions to $6.7 \pm 1.7 \%$ in the presence of DHF $(n=4)$. Also in this case, enzyme inactivation was prevented by SOD $(1,000 \mathrm{U} / \mathrm{ml})$, as conversion averaged $52.4 \pm 3.7 \%$. Similar results were obtained when LDL were exposed to $10 \mu \mathrm{M} \mathrm{CuSO}_{4}\left(1 \mathrm{~h}\right.$ at $37^{\circ} \mathrm{C}$ ) (data not shown). The similarity of the effects with three unrelated systems, and their prevention by SOD, rules out that loss of enzyme activity could have been due to accumulation of uric acid (formed from xanthine oxidation), to possible changes in the $\mathrm{pH}$ of the reaction mixture, or to other nonspecific effects of the systems used to generate oxygen radicals.

To further characterize the oxidant species responsible for inactivation of PAF acetylhydrolase, in additional experiments LDL was exposed to xanthine/xanthine oxidase in the presence of either the hydroxyl radical scavenger DMTU (10 $\mathrm{mM})$, or the iron chelator deferoxamine $(100 \mu \mathrm{M})(n=4)$. Acetylhydrolase converted $53.5 \pm 7.8 \%$ of $\left[{ }^{3} \mathrm{H}\right] \mathrm{PAF}$ to $\left[{ }^{3} \mathrm{H}\right.$ ]lyso-PAF under control conditions and $2.4 \pm 0.6 \%$ after $3 \mathrm{~h}$ of exposure to xanthine/xanthine oxidase. Inactivation was completely prevented by either DMTU or deferoxamine, as conversion of PAF over $15 \mathrm{~min}$ averaged $45.8 \pm 8.5$ and $46.6 \pm 8.0 \%$, respectively.

Loss of PAF acetylhydrolase activity was similarly observed when whole human plasma was exposed to oxygen radicals. Under the conditions of the assay, $10 \mu$ l of control plasma from normal donors converted $39.9 \pm 5.8 \%$ of PAF to lyso-PAF over $15 \min (n=4)$. However, PAF acetylhydrolase activity was reduced to $11.3 \pm 3.4 \%$ when PAF was incubated with an identical amount of plasma that had been previously exposed to xanthine/xanthine oxidase $(200 \mu \mathrm{M}$ and $100 \mathrm{mU} / \mathrm{ml}$, respectively; $n=4$ ). This finding indicates that oxygen radical-induced inactivation of PAF acetylhydrolase was not linked to possible alterations the enzyme may have undergone during the process of purification, nor to the removal of other proteins and/or components normally present in plasma.

Time course of PAF acetylhydrolase inactivation by oxygen radicals. In the absence of oxygen radicals, LDL-associated PAF acetylhydrolase did not show loss of activity when incubated at $37^{\circ} \mathrm{C}$ for $3 \mathrm{~h}$, and only a modest decrease $(<20 \%)$ was observed when incubation was prolonged up to $18 \mathrm{~h}$, consistent with previous observations $(16,45)$. In contrast, exposure to oxygen radicals rapidly inactivated the enzyme. PAF acetylhydrolase had already lost $>50 \%$ of its activity after 60 s of incubation with xanthine/ xanthine oxidase (Fig. 2). Inactivation then continued at a slower rate and was virtually complete between 10 and $15 \mathrm{~min}$ (Fig. 2). These findings are consistent with the time course of oxygen radical generation by this system (see below). Inactivation by oxygen radicals of LDL-associated PAF acetylhydrolase was apparently an irreversible phenomenon, since in additional experiments no recovery of activity of oxygen radical-treated acetylhydrolase was observed during $18 \mathrm{~h}$ of incubation at $37^{\circ} \mathrm{C}$.

Characteristics of oxygen radical-induced inactivation of $P A F$ acetylhydrolase. The inhibitory effects of oxygen radicals on PAF acetylhydrolase activity could have resulted from a decrease in the affinity of the enzyme toward its substrate or from a reduction in the number of functionally active enzyme molecules. To differentiate between these two possibilities, in additional experiments the rate of PAF conversion by PAF acetylhydrolase was measured at various concentrations of the substrate (i.e., PAF). Consistent with classical enzyme ki-

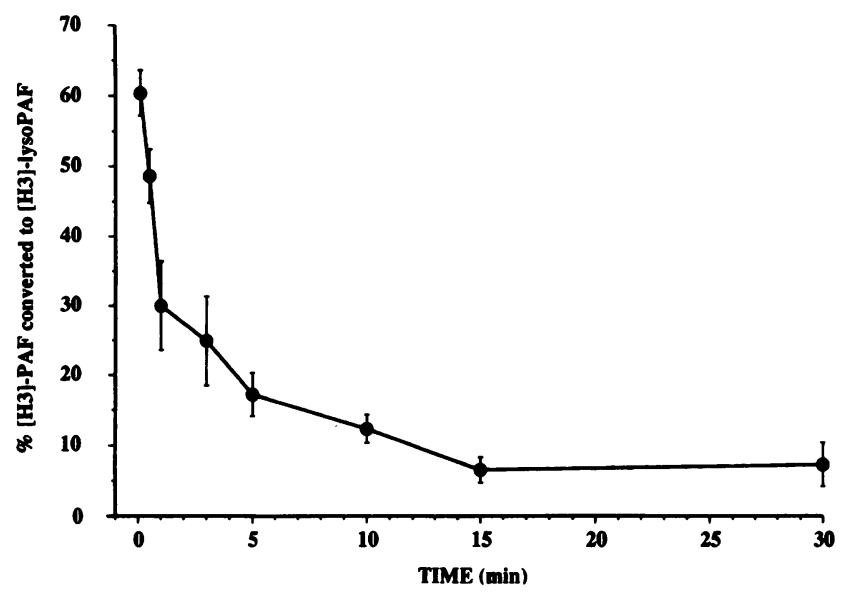

Figure 2. Time course of PAF acetylhydrolase inactivation by oxygen radicals generated in the xanthine/xanthine oxidase reaction (200 $\mu \mathrm{M}$ xanthine plus $100 \mathrm{mU} / \mathrm{ml}$ xanthine oxidase). Oxygen radicals were scavenged at the various time points by addition of SOD ( 330 $\mathrm{U} / \mathrm{ml}$ ). Each point represents the mean $\pm \mathrm{SE}$ of four separate experiments.

netics, the velocity of PAF conversion to lyso-PAF by acetylhydrolase increased sharply when the concentration of substrate in the reaction mixture was increased under nonsaturating conditions (i.e., in the $10^{-7}-10^{-5} \mathrm{M}$ range). The increase in velocity showed the expected attenuation as substrate concentration approached saturation $\left(10^{-4}-10^{-3} \mathrm{M}\right)$ (Fig. 3). From the concentration / velocity relationship of the reaction, the apparent $K_{\mathrm{m}}$ of acetylhydrolase was calculated. Under control conditions, $K_{\mathrm{m}}$ was $17.9 \mu \mathrm{M}$, consistent with previous reports (18) (Fig. 4, top). In the case of PAF acetylhydrolase exposed to oxygen radicals, a rightward shift of the concentration/velocity curve (and the consequent increase in $K_{\mathrm{m}}$ ) would indicate reduction in enzyme affinity, whereas a downward shift would be consistent with decreased number of active enzyme molecules, with no changes in affinity. When acetylhydrolase that had been exposed to oxygen radicals was analyzed, the amount of PAF converted to lyso-PAF increased progressively with increasing concentration of the substrate. The relationship closely paralleled that obtained with the control enzyme, but on a much smaller scale (Fig. 3), and the apparent $K_{\mathrm{m}}$ remained unchanged (15.1 $\mu \mathrm{M}$ ) (Fig. 4, bottom). Thus, loss of enzyme activity upon exposure to oxygen radicals was confirmed across a broad range (i.e., 1,000-fold) of substrate concentrations. The downward shift of the curve with no increase in $K_{\mathrm{m}}$ denotes that this phenomenon most likely was due to enzyme inactivation, rather than to a decrease in affinity.

We also evaluated whether loss of activity in acetylhydrolase exposed to oxidants could still be observed if we varied the length of enzyme incubation with PAF. Rate of $\left[{ }^{3} \mathrm{H}\right] \mathrm{PAF}$ conversion to $\left[{ }^{3} \mathrm{H}\right]$ lyso-PAF by xanthine oxidase-treated LDL was markedly decreased when compared with control when measured at $15 \mathrm{~min}$ of reaction (the time point used for all other experiments described in this study). A similar difference in reaction rate was observed with incubation times from 2 to $30 \mathrm{~min}$ (Table I), thus further confirming that inability to hydrolyze PAF was due to true inactivation of the enzyme.

Since free sulphydryl groups are a preferential target of oxidants, we also evaluated whether incubation with an -SH group donor, DTT, was capable of restoring the activity of PAF ace- 


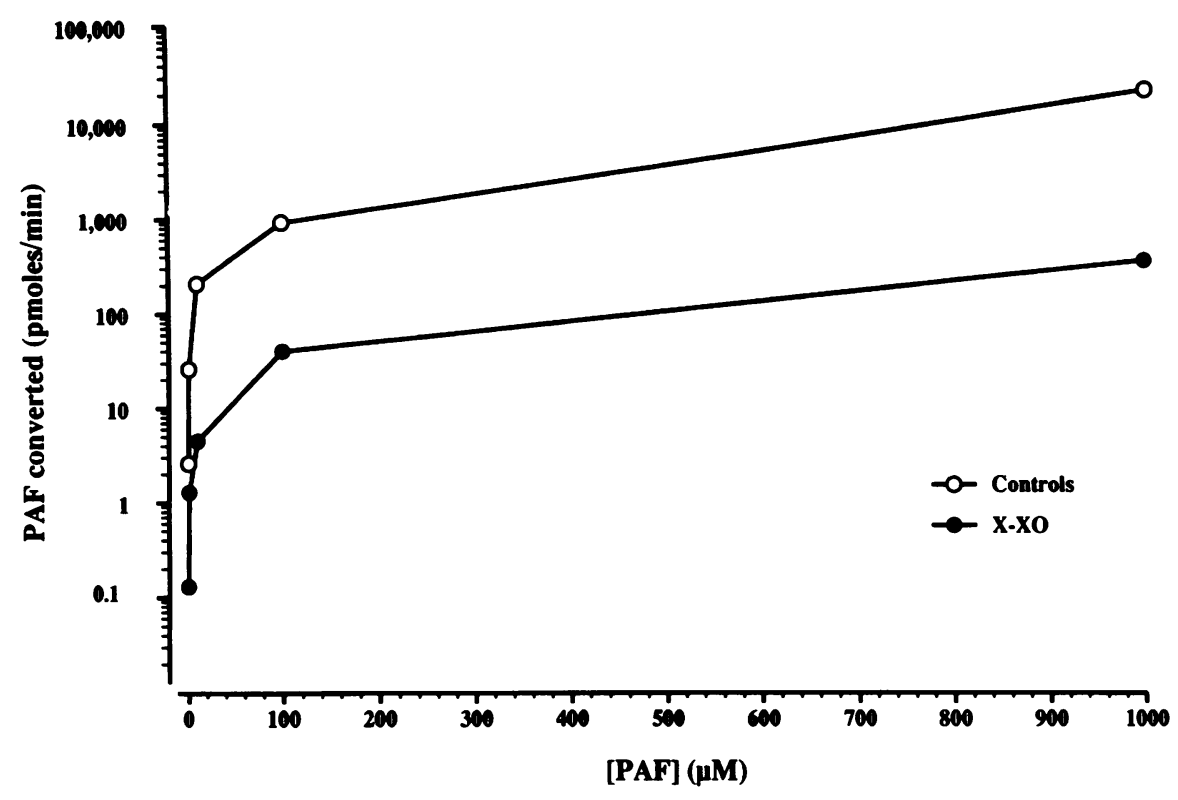

Figure 3. Effects of increasing the concentration of substrate on the velocity of acetylhydrolase-catalyzed reaction (expressed as picomoles of PAF converted per minute) of control and oxygen radical-inactivated LDL. Each point corresponds to a 10 -fold increase in PAF concentration, from $10^{-7}$ to $10^{-3} \mathrm{M}$. Exposure to xanthine/xanthine oxidase $(X-X O)$ reduced the velocity of reaction by about two orders of magnitude at all concentrations, without altering the overall shape of the curve. Each point represents the mean of three separate experiments. tylhydrolase that had been inactivated by prior exposure to oxygen radicals. Consistent with previous reports $(16,45), 3 \mathrm{~h}$ of incubation with a large excess of DTT (i.e., $10 \mathrm{mM}$ ) at $37^{\circ} \mathrm{C}$ did not affect PAF acetylhydrolase activity of control LDL. In parallel experiments, PAF acetylhydrolase activity was first inhibited (to $21 \%$ of controls) by exposing LDL to xanthine/ xanthine oxidase $(200 \mu \mathrm{M}$ and $100 \mathrm{mU} / \mathrm{ml}$, respectively, for 3 $\mathrm{h}$ at $\left.37^{\circ} \mathrm{C} ; n=4\right)$. Then, aliquots were incubated for an additional $3 \mathrm{~h}$ with $10 \mathrm{mM}$ DTT at $37^{\circ} \mathrm{C}$. DTT failed to restore PAF acetylhydrolase activity, which remained at $23 \%$ of controls $(n=4)$.

To evaluate whether the presence of substrate could protect acetylhydrolase from inactivation by oxygen radicals, in additional experiments LDL were exposed to the xanthine/xanthine oxidase system in the presence of a large amount of unlabeled PAF $\left(10^{-5} \mathrm{M}\right)$. Incubation with xanthine/xanthine oxidase abolished PAF acetylhydrolase activity, both under standard conditions ( to $2 \%$ of control; $n=4$ ), as well as when PAF was present before and during oxygen radical generation (to $3 \% ; n=4$ ). Lack of a protective effect from the substrate suggests that the inhibitory effects of oxygen radicals were not secondary to modification of the enzyme at (or near) the active site.

LDL may undergo major modifications when exposed to oxidants, characterized by fragmentation of apoprotein, markedly increased electrophoretic mobility, and extensive peroxidation of lipid moiety $(33,34,46-48)$. Therefore, specific experiments were performed to investigate whether loss of PAF acetylhydrolase activity was secondary to oxidative modifications of LDL. Exposure to xanthine/xanthine oxidase for $\mathbf{3 0}$ min did not induce aggregation or fragmentation of apoprotein, as judged from polyacrylamide gel electrophoresis under denaturating conditions (Fig. 5, left panel). Similarly, electrophoretic mobility on agarose gel was minimally or not increased in LDL exposed to oxygen radicals under our experimental conditions (Fig. 5, right panel), even though enzyme activity was reduced to $<10 \%$ of control. Thus, modifications of LDL protein are unlikely to account for the observed loss of PAF acetylhydrolase activity. Finally, we checked whether xanthine/xanthine oxidase treatment would induce LDL lipid peroxidation. LDL were incubated with the oxygen radicalgenerating system and analyzed after $15 \mathrm{~min}, 30 \mathrm{~min}$, or $18 \mathrm{~h}$ for malonyldialdehyde content as a marker of lipid peroxidation. At 15 or 30 min of incubation, a time largely sufficient to induce inactivation of PAF acetylhydrolase, no significant increase was observed in the concentration of lipid peroxidation products over the small values found in control LDL (Fig. 6). Prolonging the length of incubation to $18 \mathrm{~h}$ was associated with a > 10-fold increase in malonyldialdehyde concentrations, similar to what was observed in previous studies $(34,46-48)$. This phenomenon, however, could be still blocked by adding the inhibitor of lipid peroxidation butylated hydroxytoluene ( 50 $\mu \mathrm{M}) 30 \mathrm{~min}$ after the xanthine oxidase reaction had started (Fig. 6), i.e., when loss of enzyme activity had already occurred. Taken together, these findings demonstrate that, under our conditions, inactivation of PAF acetylhydrolase by oxygen radicals was temporally distinct from development of peroxidation of LDL lipids.

Effect of different concentrations of oxygen radicals on $P A F$ acetylhydrolase activity. In another set of experiments, we tested the effects of different concentrations of the oxygen radical-generating system on PAF acetylhydrolase activity. Three conditions were used (Fig. 7, left): (a) xanthine $200 \mu \mathrm{M}$, xanthine oxidase $100 \mathrm{mU} / \mathrm{ml}$ : this was the concentration also used in the experiments reported above and generated a total amount of superoxide radicals of $\sim 50 \mathrm{nmol}$, which peaked at $\sim 20 \mathrm{nmol} / \mathrm{min}$ per $\mathrm{ml}$ within $60 \mathrm{~s}$ and rapidly declined within $5 \mathrm{~min}$; (b) xanthine $200 \mu \mathrm{M}$, xanthine oxidase $50 \mathrm{mU} / \mathrm{ml}$ : with these concentrations, peak superoxide radical production was $\sim 10 \mathrm{nmol} / \mathrm{min}$ per $\mathrm{ml}$, and total production was about two thirds of $a$; (c) xanthine $20 \mu \mathrm{M}$, xanthine oxidase $50 \mathrm{mU}$ : this system also yielded a lower peak concentration as compared with $a$ for a shorter duration of time and with a further decrease of total production (about one half of $a$ ).

When tested on LDL-associated PAF acetylhydrolase, the three oxygen radical-generating systems affected enzyme activity in a dose-dependent fashion (Fig. 7, right panel). As already shown, PAF acetylhydrolase activity was almost completely lost upon incubation with $200 \mu \mathrm{M}$ xanthine plus $100 \mathrm{mU} / \mathrm{ml}$ of xanthine oxidase. Enzyme activity was still reduced signifi- 

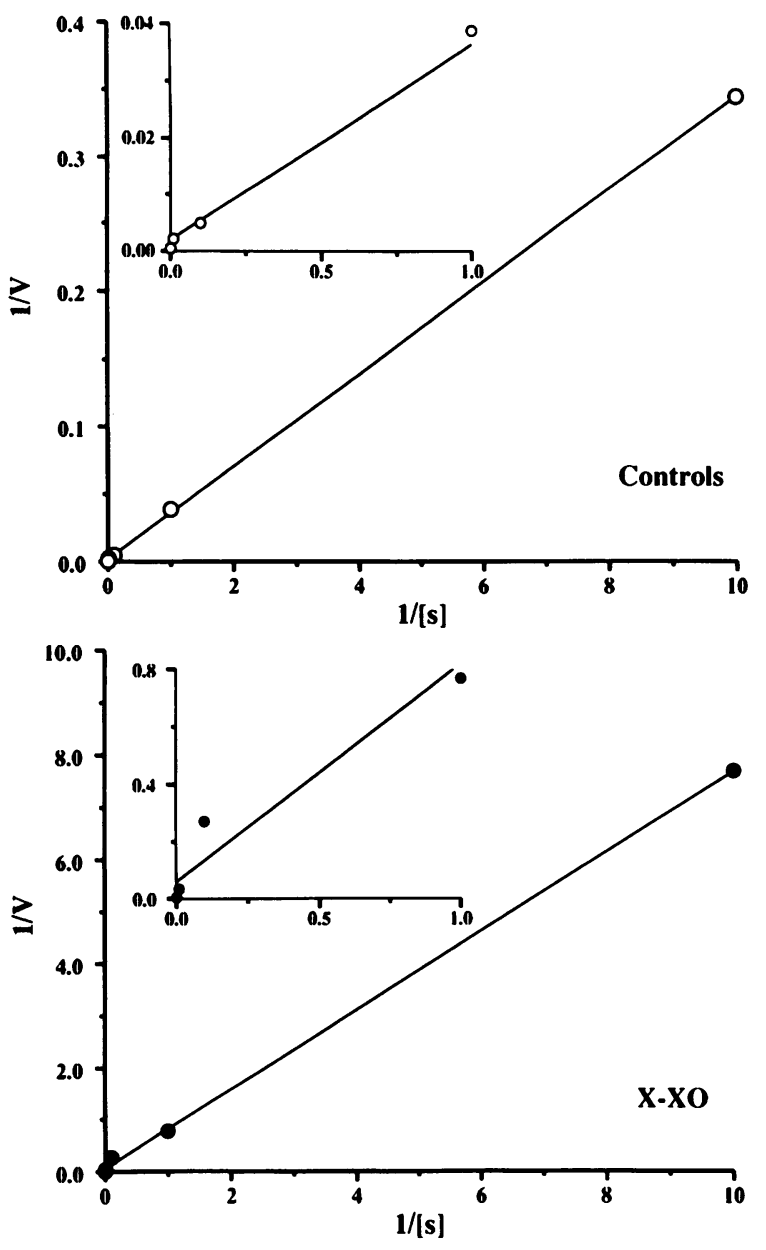

Figure 4. (Top) Substrate-velocity relationship of PAF acetylhydrolase under control conditions according to Lineweaver-Burk analysis. The reciprocal of velocity is plotted as a function of the reciprocal of substrate concentration for the five experimental points reported in Fig. 3. The data fit the linear equation: $y=3.42 \cdot 10^{-2}$ $X+1.92 \cdot 10^{-3} ; r^{2}=0.99$. The $K_{\mathrm{m}}$ of the enzyme is estimated at $17.9 \mu \mathrm{M}$. The inset shows on an expanded scale the relationship for the last four points of the curve. (Bottom) Substrate-velocity relationship of PAF acetylhydrolase after exposure to oxygen radicals according to Lineweaver-Burk analysis. Format as in the above panel, with a different $y$-axis scale. The data fit the linear equation: $y$ $=7.63 \cdot 10^{-1} \times+5.06 \cdot 10^{-2} ; r^{2}=0.99$. The $K_{\mathrm{m}}$ of the enzyme is estimated at $15.1 \mu \mathrm{M}$.

cantly in LDL that had been exposed to a $30 \%$ lower concentration of superoxide radicals. A trend toward enzyme inactivation was also observed when LDL were incubated with the lowest concentration of radicals, although this difference did not reach statistical significance.

Table I. Kinetics of PAF Hydrolysis by Acetylhydrolase

\begin{tabular}{llllll}
\hline & $2 \mathrm{~min}$ & $4 \mathrm{~min}$ & $8 \mathrm{~min}$ & $16 \mathrm{~min}$ & $32 \mathrm{~min}$ \\
\hline Control & 0.23 & 0.22 & 0.23 & 0.21 & 0.12 \\
$\mathrm{X} / \mathrm{XO}$ & 0.014 & 0.015 & 0.012 & 0.012 & 0.015
\end{tabular}

Results (nanomoles of PAF converted per minute) are the results of duplicate experiments. $X / X O$, acetylhydrolase exposed to xanthine/xanthine oxidase for $3 \mathrm{~h}$ at $37^{\circ} \mathrm{C}$.

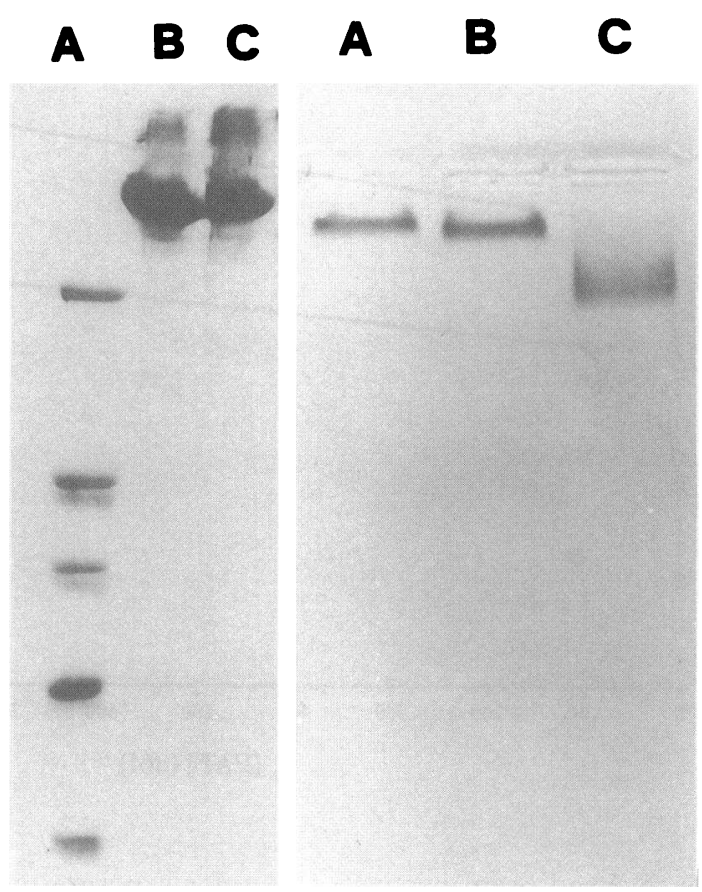

Figure 5. (Left panel) Polyacrylamide gel electrophoresis under denaturing conditions ( $1 \%$ SDS) of control LDL (lane $B$ ) and LDL that has been exposed to xanthine/xanthine oxidase for $30 \mathrm{~min}$. Lane $A$ shows molecular weight standards. (Right panel) Agarose gel electrophoresis of control LDL (lane $A$ ), LDL exposed to xanthine/xanthine oxidase for $30 \mathrm{~min}$, and $\mathrm{LDL}$ oxidized by $\mathrm{CuSO}_{4}(10 \mu \mathrm{M}$ for $18 \mathrm{~h}$ ). PAF acetylhydrolase activity of both forms of oxidized LDL was $<5 \%$ of control.

\section{Discussion}

In this study, exposure of whole human plasma or purified LDL to a source of oxygen radicals resulted in marked loss of PAF acetylhydrolase activity. This phenomenon was rapid and

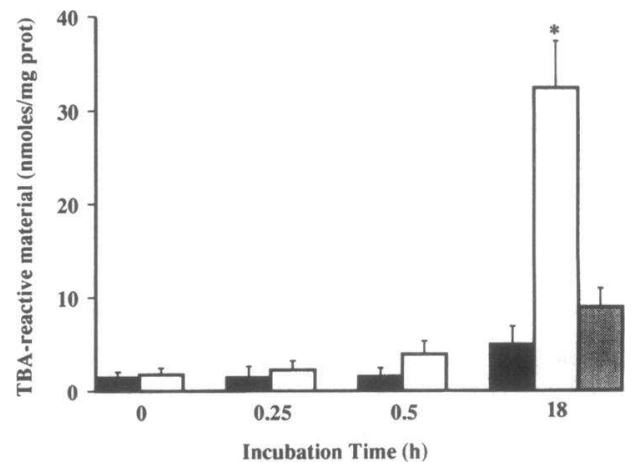

Figure 6. Concentration of lipid peroxidation products, measured as thiobarbituric acid-reactive material $(T B A)$ in LDL incubated for various times at $37^{\circ} \mathrm{C}$ under control conditions (solid bars), or in the presence of the oxygen radical-generating system xanthine/xanthine oxidase (X/XO) (open bars). Stipled bar shows the effects on TBAreactive material formation of the addition of the lipid peroxidation inhibitor butylated hydroxytoluene $(50 \mu \mathrm{M})$ added $30 \mathrm{~min}$ after the xanthine oxidase reaction had started. Results are the mean \pm SE of five experiments. PAF acetylhydrolase activity averaged $11 \pm 4 \%$ of control in LDL exposed to X/XO for $15 \mathrm{~min}$ and $5 \pm 3 \%$ in LDL exposed for $30 \mathrm{~min}$. 

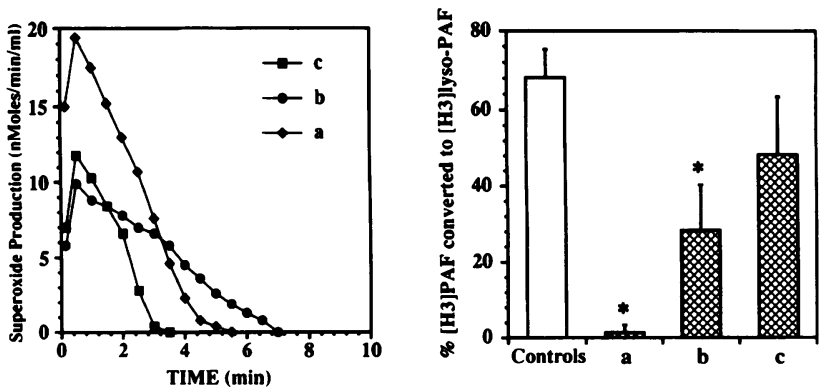

Figure 7. (Left panel) Time course of superoxide radical production by different concentrations of the xanthine/xanthine oxidase system. (a) $200 \mu \mathrm{M}$ xanthine plus $100 \mathrm{mU} / \mathrm{ml}$ xanthine oxidase; (b) $200 \mu \mathrm{M}$ xanthine plus $50 \mathrm{mU} / \mathrm{ml}$ xanthine oxidase; (c) $20 \mu \mathrm{M}$ xanthine plus $50 \mathrm{mU} / \mathrm{ml}$ xanthine oxidase (see text for details). (Right panel) Effects of exposure to varying amounts of superoxide radicals on PAF acetylhydrolase activity. Enzyme activity was measured on control LDL, as well as on LDL exposed to one of the three xanthine/ xanthine oxidase systems depicted in the left panel $(n=5$ for each group). *Significantly different from controls $(P<0.05)$.

irreversible, and, since it was prevented by the scavenger enzyme SOD and other anti-free radical agents, it was a specific consequence of oxygen radical generation. In vivo, several pathophysiological processes may result in the concomitant formation of PAF and oxygen radicals during tissue injury. Thus, inactivation of acetylhydrolase, the enzyme that catabolyzes PAF, might be one mechanism by which oxygen radicals may potentiate the important proinflammatory effects of this mediator.

Oxygen radicals are chemical species produced by all aerobic organisms characterized by the presence of an unpaired electron, which renders them extremely reactive. Exposure to oxygen radicals results in rapid inactivation, among others, of creatinekinase (29), glutathione peroxidase (30), and cyclooxygenase (31). While the precise requisites which favor protein modification by oxygen radicals are only partially clarified, amino acids with double bonds or free - $\mathrm{SH}$ groups are candidates for oxygen radical attack (28). It has been proposed that inactivation by oxygen radicals might represent one mechanism regulating the activity of several enzyme systems. Cyclooxygenase, the key enzyme of prostaglandin metabolism, is perhaps the best known example of this phenomenon. This enzyme catalyzes two different reactions: oxygenation of arachidonic acid to $\mathrm{PGG}_{2}$ and the subsequent conversion of $\mathrm{PGG}_{2}$ to $\mathrm{PGH}_{2}$. This latter reaction is accompanied by formation of oxygen radicals, which in turn irreversibly inactivate cyclooxygenase $(31,49)$. It is currently believed that this "suicidal" inactivation is in fact a major mechanism controlling prostanoid production in vivo (50).

In the present study, inactivation of PAF acetylhydrolase was achieved upon exposure of the enzyme to xanthine/ xanthine oxidase or to DHF. These are two well characterized oxygen radical-generating systems, chemically unrelated to each other, that are widely used to investigate the effects of oxygen radicals under controlled conditions $(36,37,43,44)$. Our data provide a partial characterization of the effects of oxygen radicals on human PAF acetylhydrolase. Oxygen radical-induced inactivation was observed both on whole plasma and on purified LDL. This phenomenon occurred in a dose-dependent fashion, was rapid and irreversible, and did not in- volve changes in the affinity of acetylhydrolase toward its substrate PAF. Our data indicate that loss of PAF acetylhydrolase activity upon exposure to oxygen radicals was not the consequence of oxidative changes of LDL itself, and that modifications of amino acid residues at (or near) the active site of the enzyme or oxidation of free -SH groups were unlikely to account for the phenomenon. Further purification of the enzyme was not attempted, since it has been shown that the acetylhydrolase activity responsible for PAF hydrolysis in plasma is associated with LDL $(17,51)$. Furthermore, because of its highly hydrophobic nature, plasma PAF acetylhydrolase represents an integral component of LDL particles, from which it can be separated only under nonphysiologic conditions (i.e., in the presence of detergents and/or denaturing agents [15-17]).

The results of the various experiments performed with different anti-free radical agents show that inactivation of PAF acetylhydrolase could be prevented completely by SOD, by the hydroxyl radical scavenger DMTU, or by the iron chelator deferoxamine. These results are consistent with the known mechanism of hydroxyl radical formation by superoxide radicals, in an iron-catalyzed reaction (39). Metal-catalyzed formation of hydroxyl radicals has been shown to account for selective, "site-specific" inactivation of several enzymes (28). Thus, our data indicate that hydroxyl radical is the oxidant species ultimately responsible for PAF acetylhydrolase inactivation and that the phenomenon can be blocked by a hydroxyl radical scavenger. However, they also show that a similar protective effect can be achieved if one prevents hydroxyl radicals from being formed, by removing the initial trigger of the reaction (i.e., superoxide radical) or the catalyst (i.e., iron). Superoxide-driven hydroxyl radical formation also requires peroxides. We found that catalase, a scavenger of hydrogen peroxide, was ineffective in preventing xanthine oxidase-induced inactivation of acetylhydrolase. One possible explanation for this failure is that, because of its relatively high $K_{\mathrm{m}}$, catalase did not reduce $\mathrm{H}_{2} \mathrm{O}_{2}$ concentrations to a low enough level to block formation of hydroxyl radicals. Alternatively, it may be speculated that the reaction might have still occurred even in the absence of hydrogen peroxide, supported by lipid peroxides that we and others found in control LDL, presumably as a consequence of some degree of spontaneous oxidation occurring in vivo and/or during the isolation procedures (Fig. 6) $(34,46,47)$.

In spite of the importance of acetylhydrolase in regulating PAF concentrations, little is known with regard to the effects of pathophysiologically relevant conditions on the activity of this enzyme. Earlier studies only measured changes in acetylhydrolase levels of plasma in experimental models of hypertension (52) or pregnancy (53), or in patients with asthma (54). No previous study has investigated the effects of an intervention on the activity of PAF acetylhydrolase, with the exception of a recent report by Miyaura et al. (45), who showed that acetylhydrolase activity of whole plasma was inhibited by a cigarette smoke extract. However, that study differs from ours in several important aspects, since it was not performed on purified LDL and the factor(s) responsible for enzyme inhibition were not identified. Furthermore, acetylhydrolase inhibition by smoke extract occurred over several hours, and oxygen radicals were apparently not involved in the phenomenon. Indirect support to our results may derive from earlier reports by other investigators, who observed that LDL-associated acetylhydrolase activity was labile under oxidant conditions $(55,56)$. However, 
in those experiments the phenomenon was induced by other oxidant systems, and it was much less pronounced than in our study (56) or it was not characterized (55). Thus, the present study is the first direct demonstration that important mediators of tissue injury, such as superoxide and hydroxyl radicals, can importantly affect the activity of the enzyme responsible for the metabolism of the proinflammatory mediator PAF.

Our findings are limited to observations obtained in vitro. However, it is conceivable that inactivation of PAF acetylhydrolase by oxygen radicals may also occur in vivo. This speculation is based on the following considerations. First, we show that the phenomenon is not restricted to purified LDL but can be similarly observed when whole plasma is exposed to radicals, thus indicating that inactivation can still occur in spite of possible endogenous antioxidants present in plasma. Second, the rate of superoxide radical generation to which acetylhydrolase has been exposed in our study $(10-20 \mathrm{nmol} / \mathrm{min}$ per $\mathrm{ml})$ is within the range that can be encountered in vivo. Unstimulated neutrophils produce about $1 \mathrm{nmol}$ of superoxide/min per million cells and respond to stimulation with a 10-20-fold increase that lasts several minutes $(57,58)$. Furthermore, oxygen radical concentration at the site of inflammation can be much higher, for the combined effects of neutrophil accumulation and activation. Oxygen radicals can also be formed by other mechanisms, including mitochondrial respiration (59) and activation of resident cells $(22,60,61)$, which may also be operative under conditions of tissue injury and which may add to the total radical production. Finally, direct electron paramagnetic resonance experiments have measured concentrations of oxygen radicals averaging 8-10 $\mu \mathrm{M}$ during reperfusion of ischemic hearts $(19,20,62,63)$. This concentration of oxygen radicals and its attendant toxic effect can be markedly ablated by administration of SOD, deferoxamine, or hydroxyl radical scavengers $(20,62-64)$. Thus, it is conceivable that superoxide-driven, iron-mediated inactivation of PAF acetylhydrolase by hydroxyl radicals may occur in this setting. In this respect, clinical data (65) show that plasma PAF acetylhydrolase activity is reduced in patients undergoing cardiac arrest and reperfusion during open-heart surgery, a condition likely to be associated with oxygen radical production $(66,67)$.

The finding that oxygen radicals can inactivate PAF acetylhydrolase might have potentially important implications. As already pointed out, both PAF and oxygen radicals are produced by activated cells (e.g., neutrophils, macrophages, mast cells, and endothelial cells) in a variety of pathophysiological conditions. After the initial damage, tissue injury is maintained and amplified by several mechanisms, in which PAF and oxygen radicals can each play an important and independent role. However, the results of this study suggest that, in addition to inducing direct tissue damage in their own right, oxygen radicals might also potentiate the inflammatory effects of PAF secondary to inactivation of acetylhydrolase. Higher (and/or longer lasting) concentrations of PAF may then be expected to result in further accumulation and enhanced activation of neutrophils and macrophages $(4,60)$. As a consequence, additional PAF and oxygen radicals will be generated, thus providing the setting for possible amplification and perpetuation of the inflammatory reaction. The hypothesis that oxygen radicals might indirectly potentiate PAF toxicity may provide an explanation to recent observations. In a model of intestinal ischemia/reperfusion, Granger and associates $(23,24,68)$ have shown that vascular damage is largely the consequence of
PAF and oxygen radical production by activated neutrophils and resident phagocytic cells. Those effects were not independent of each other, as these authors demonstrated that PAF-induced increase in mucosal permeability was blunted by administration of oxygen radical scavengers $(23,24,68)$ and that SOD reduced neutrophil adherence to the endothelium (23, 68 ). To explain these findings, they postulated that superoxide radical production might have enhanced the effects of PAF (68). Similar results have been obtained by other investigators (69). Reperfusion of the ischemic heart might be another example. Here, too, the stage is set for production of oxygen radicals and PAF upon reflow $(19,20,70)$, which is followed by marked influx of neutrophils (71). Of interest, it has been shown that PAF may propagate myocardial ischemic damage (13) and that hydrolysis by acetylhydrolase is the major route of PAF degradation in the heart (72). Another condition where oxygen radicals and PAF might potentiate the effects of each other is bronchial asthma. On the one hand, PAF is considered an important mediator of asthma $(5,10)$. On the other hand, clinical and experimental studies link together increased oxygen radical production and enhanced bronchial constriction to various stimuli (25-27), even though radicals apparently have no direct effects on bronchial tone $(25)$. It is possible that a similar interrelationship between oxygen radicals and PAF might also occur in other conditions. In this respect, it is noteworthy that SOD and other antioxidants may show antiinflammatory properties (73-76) and, conversely, that many antiinflammatory or antiallergy drugs have demonstrated oxygen radical-scavenging properties $(76,77)$.

In conclusion, the results of the present study provide a biochemical basis for a possible interplay between PAF and oxygen radicals by documenting that acetylhydrolase, the enzyme that catabolizes PAF, can be inactivated by oxygen radicals. Oxygen radicals and PAF are both formed at the site of inflammation. PAF is known to stimulate several cell types, with further enhancement of PAF production and oxygen radical formation. If this phenomenon were also accompanied by concomitant inactivation of acetylhydrolase, it would contribute to maintaining and amplifying the inflammatory effects of PAF.

\section{Acknowledgments}

This study was supported in part by grants 91.00122.PF41 and 92.00016.PF41 (Progetto Finalizzato Prevenzione e Controllo dei Fattori di Malattia) and 92.01230 (Progetto Finalizzato Biotecnologie e Biostrumentazioni) from Consiglio Nazionale delle Ricerche, Rome, Italy. This work has been conducted during the tenure of Dr. Claudio Napoli as a Postdoctoral Fellow of the Italian Ministry of Health (047.91).

\section{References}

1. Henson, P. M. 1970. Release of vasoactive amines from rabbit platelets induced by sensitized mononuclear leukocytes and antigen. J. Exp. Med. 131:287-304.

2. Benveniste, J., P. M. Henson, and C. G. Cochrane. 1972. Leukocyte-dependent histamine release from rabbit platelets. The role of IgE, basophils, and a platelet-activating factor. J. Exp. Med. 136:1356-1377.

3. Demopoulos, C. A., R. N. Pinckard, and D. J. Hanahan. 1980. Platelet-activating factor (PAF). Evidence for 1-O-alkyl-2-acetyl-sn-glyceryl-3-phosphorylcholine as the active component. A new class of lipid chemical mediators. J. Biol. Chem. 254:9355-9358.

4. Shaw, J. O., R. N. Pinckard, K. S. Ferrigni, L. M. McManus, and D. J. Hanahan. 1981. Activation of human neutrophils with 1-O-hexadecyl/octade- 
cyl-2-acetyl-sn-glyceryl-3-phosphorylcholine (platelet-activating factor). J. Immunol. 127:1250-1255.

5. Hanahan, D. J. 1986. Platelet activating factor: a biologically active phosphoglyceride. Annu. Rev. Biochem. 55:483-509.

6. Laurindo, F. R. M., R. E. Goldstein, N. J. Davenport, D. Ezra, and G. Z. Feuerstein. 1989. Mechanism of hypotension produced by platelet-activating factor. J. Appl. Physiol. 66:2681-2690.

7. Jackson, C. V., W. A. Schumacher, S. L. Kunkel, E. M. Driscoll, and B. R. Lucchesi. 1986. Platelet-activating factor and the release of a platelet-derived coronary artery vasodilator substance in the canine. Circ. Res. 58:218-229.

8. Kenzora, J. L., J. E. Perez, S. R. Bergmann, and L. G. Lange. 1984. Effects of acetyl glyceryl ether of phosphorylcholine (platelet-activating factor) on ventricular preload, afterload, and contractility in dogs. J. Clin. Invest. 74:11931203.

9. Robertson, D. A., A. Genovese, and R. Levi. 1987. Negative inotropic effect of platelet-activating factor on human myocardium: a pharmacological study. J. Pharmacol. Exp. Ther. 243:834-839.

10. Braquet, P., L. Touqui, T. Y. Shen, and B. B. Vargaftig. 1987. Perspectives in platelet-activating factor research. Pharmacol. Rev. 39:97-146.

11. Kubes, P., G. Ibbotson, J. Russel, J. L. Wallace, and D. N. Granger. 1990. Role of platelet-activating factor in ischemia/reperfusion-induced leukocyte adherence. Am. J. Physiol. 259:G300-G305.

12. Doebber, T. W., M. S. Wu, J. C. Robbins, M. Choy, M. N. Chang, and T. Y. Shen. 1985. Platelet activating factor (PAF) involvement in endotoxin-induced hypotension in rats. Studies with PAF-receptor antagonist kadsurenone. Biochem. Biophys. Res. Commun. 127:799-808.

13. Stahl, G. L., Z. I. Terashita, and A. M. Lefer. 1988. Role of platelet-activating factor in propagation of cardiac damage during myocardial ischemia. $J$. Pharmacol. Exp. Ther. 244:898-904.

14. Wallace, J. L., and B. J. R. Whittle. 1986. Picomole doses of platelet-activating factor predispose the gastric mucosa to damage by topical irritants. Prostaglandins. 31:989-998.

15. Farr, R. S., C. P. Cox, M. L. Wardlow, and R. Jorgensen. 1980. Preliminary studies of an acid-labile factor (ALF) in human sera that inactivates plateletactivating factor (PAF). Clin. Immunol. Immunopathol. 15:318-330.

16. Wardlow, M. L., C. P. Cox, K. E. Meng, D. E. Greene, and R. S. Farr. 1986. Substrate specificity and partial characterization of the PAF-acetylhydrolase in human serum that rapidly inactivates platelet-activating factor. $\mathrm{J}$. Immunol. 136:3441-3446.

17. Stafforini, D. M., T. M. McIntyre, M. E. Carter, and S. M. Prescott. 1987. Human plasma platelet-activating factor acetylhydrolase. Association with lipoprotein particles and role in the degradation of platelet-activating factor. J. Biol. Chem. 262:4215-4222.

18. Stafforini, D. M., S. M. Prescott, and T. M. McIntyre. 1987. Human plasma platelet-activating factor acetylhydrolase. Purification and properties. $J$. Biol. Chem. 262:4223-4230.

19. Zweier, J. L., J. T. Flaherty, and M. L. Weisfeldt. 1987. Direct measurement of free radical generation following reperfusion of ischemic myocardium. Proc. Natl. Acad. Sci. USA. 84:1404-1407.

20. Bolli, R., B. S. Patel, M. O. Jeroudi, E. K. Lai, and P. B. McCay. 1988. Demonstration of free radical generation in "stunned" myocardium of intact dogs with the use of the spin trap alpha-phenyl $N$-Tert-butyl nitrone. J. Clin. Invest. 82:476-485.

21. Fantone, J. C., and P. A. Ward. 1982. Role of oxygen-derived free radicals and metabolites in leukocyte-dependent inflammatory reactions. Am. J. Pathol. 107:397-418.

22. Nathan, C. F. 1987. Secretory products of macrophages. J. Clin. Invest. 79:319-326.

23. Suzuki, M., W. Inauen, P. R. Kvietys, M. B. Grisham, C. Meininger, M. E. Schelling, H. J. Granger, and D. N. Granger. 1989. Superoxide mediates reperfusion-induced leukocyte-endothelial cell interactions. Am. J. Physiol. 257:H1740H1745.

24. Kubes, P., K. E. Arfors, and D. N. Granger. 1991. Platelet-activating factor-induced mucosal dysfunction: role of oxidants and granulocytes. $\mathrm{Am}$. J Physiol. 260:G965-G971.

25. Postma, D. S., T. E. J. Renkema, J. A. Noordhoe, H. Faber, H. J. Sluite and H. Kauffman. 1988. Association between nonspecific bronchial hyperreactivity and superoxide anion production by polymorphonuclear leukocytes in chronic airflow obstruction. Am. Rev. Respir. Dis. 137:57-61.

26. Ikuta, N., S. Sugiyama, K. Takagi, T. Satake, and T. Ozawa. 1992. Implication of oxygen radicals on airway hyperresponsiveness after ovalbumin challenge in guinea pigs. Am. Rev. Respir. Dis. 145:561-565.

27. Kanazawa, H., N. Kurihara, K. Hirata, and T. Takeda. 1991. The role of free radicals in airway obstruction in asthmatic patients. Chest. 100:1319-1322.

28. Stadtman, E. R. 1992. Protein oxidation and aging. Science (Wash. DC). 257:1220-1224.

29. Santoro, G., G. Ambrosio, P. P. Elia, C. Duilio, G. Riccio, I. Tritto, and M. Chiariello. 1989. Inactivation of creatinekinase by superoxide radicals. $J$. Mol. Cell. Cardiol. 21:S63a. (Abstr.)
30. Blum, J., and I. Fridovich. 1985. Inactivation of glutathione peroxidase by superoxide radical. Arch. Biochem. Biophys. 240:500-508.

31. Kukreja, R. K., H. A. Kontos, M. L. Hess, and E. F. Ellis. 1986. PGH synthase and lipoxygenase generate superoxide in the presence of NADH or NADPH. Circ. Res. 59:612-619.

32. Hatch, F. T., and R. S. Lees. 1968. Plasma lipoprotein analysis. Adv. Lipid Res. 6:1-68.

33. Schuh, J., G. F. Fairclough, Jr., and R. H. Haschemeyer. 1978. Oxygenmediated heterogeneity of apo-low-density lipoprotein. Proc. Natl. Acad. Sci. USA. 75:3173-3177.

34. Sparrow, C. P., S. Parthasarathy, and D. Steinberg. 1988. Enzymatic modification of low density lipoprotein by purified lipoxygenase plus phospholipase $A_{2}$ mimics cell-mediated oxidative modification. J. Lipid Res. 29:745-753.

35. Ambrosio, G., J. T. Flaherty, C. Duilio, I. Tritto, G. Santoro, P. P. Elia, M. Condorelli, and M. Chiariello. 1991. Oxygen radicals generated at reflow induce peroxidation of membrane lipids in reperfused hearts. J. Clin. Invest. 87:20562066.

36. Lowry, O. H., H. J. Rosebrough, A. L. Farr, and R. J. Randall. 1951. Protein measurement with the Folin phenol reagent. J. Biol. Chem. 193:265275.

37. McCord, J. M., and I. Fridovich. 1968. The reduction of cytochrome $\mathrm{c}$ by milk xanthine oxidase. J. Biol. Chem. 243:5753-5760.

38. Porras, A. G., J. S. Olson, and G. Palmer. 1981. The reaction of reduced xanthine oxidase with oxygen. J. Biol. Chem. 256:9096-9103.

39. Aust, S. D., L. A. Morehouse, and C. E. Thomas. 1985. Role of metals in oxygen radical reactions. J. Free Radicals Biol. \& Med. 1:3-25.

40. Triggiani, M., and F. H. Chilton. 1989. Influence of immunologic activation and cellular fatty acid levels on the catabolism of platelet-activating factor within the murine mast cell (PT-18). Biochim. Biophys. Acta. 1006:41-51.

41. Bligh, E. G., and W. J. Dyer. 1959. A rapid method of total lipid extraction and purification. Can. J. Biochem. Physiol. 37:911-917.

42. Triggiani, M., D. M. D'Souza, and F. H. Chilton. 1991. Metabolism of 1-acyl-2-acetyl-sn-glycero-3-phosphocholine in the human neutrophil. J. Biol. Chem. 266:6928-6935.

43. Tong Mak, I., H. P. Misra, and W. B. Weglicki. 1983. Temporal relationship of free radical induced lipid peroxidation and loss of latent enzyme activity in highly enriched hepatic lysosomes. J. Biol. Chem. 258:13733-13737.

44. Cerbai, E., G. Ambrosio, F. Porciatti, M. Chiariello, A. Giotti, and A. Mugelli. 1991. Cellular electrophysiological basis for oxygen radical-induced arrythmias. A patch-clamp study in Guinea pig ventricular myocytes. Circulation. 84:1773-1782.

45. Miyaura, S., H. Eguchi, and J. M. Johnston. 1992. Effect of a cigarette smoke extract on the metabolism of the proinflammatory autocoid, platelet-activating factor. Circ. Res. 70:341-347.

46. Heinecke, J. W., L. Baker, H. Rosen, and A. Chait. 1986. Superoxide-mediated modification of low density lipoprotein by arterial smooth muscle cells. $J$. Clin. Invest. 77:757-761.

47. Jessup, W., S. M. Rankin, C. V. De Whalley, J. R. S. Hoult, J. Scott, and D. S. Leake. 1990. $\alpha$-Tocopherol consumption during low-density-lipoprotein oxidation. Biochem. J. 265:399-405.

48. Esterbauer, H., H. Puhl, M. Dieber-Rotheneder, G. Waeg, and H. Rabl 1991. Effects of antioxidants on oxidative modifications of LDL. Ann. Med. 23:573-581.

49. Lambeir, A. M., C. M. Markey, H. B. Dunford, and L. J. Marnett. 1985. Spectral properties of the higher oxidation states of prostaglandin $\mathrm{H}$ synthase. $J$. Biol. Chem. 260:14894-14896.

50. Marshall, P. J., R. J. Kulmacz, and W. E. M. Lands. 1987. Constraints on prostaglandin biosynthesis in tissues. J. Biol. Chem. 262:3510-3517.

51. Stafforini, D. M., M. E. Carter, G. A. Zimmerman, T. M. McIntyre, and S. M. Prescott. 1989. Lipoproteins alter the catalytic behavior of the platelet-activating factor acetylhydrolase in human plasma. Proc. Natl. Acad. Sci. USA 86:2393-2397.

52. Blank, M. L., M. N. Hall, E. A. Cress, and F. Snyder. 1983. Inactivation of 1-alkyl-2-acetyl-sn-glycero-3-phosphocholine by a plasma acetylhydrolase: higher activities in hypertensive rats. Biochem. Biophys. Res. Commun. 113:666671.

53. Maki, N., D. R. Hoffman, and J. M. Johnston. 1988. Platelet-activating factor acetylhydrolase activity in maternal, fetal, and newborn rabbit plasma during pregnancy and lactation. Proc. Natl. Acad. Sci. USA. 85:728-732.

54. Miwa, M., T. Miyake, T. Yamanaka, J. Sugatani, Y. Suzuki, S. Sakata, F. Araki, and M. Matsumoto. 1988. Characterization of serum platelet-activating factor (PAF) acetylhydrolase. Correlation between deficiency of serum PAF acetylhydrolase and respiratory symptoms in asthmatic children. J. Clin. Invest. 82:1983-1991.

55. Steinbrecher, U. P., and P. H. Pritchard. 1989. Hydrolysis of phosphatidylcholine during LDL oxidation is mediated by platelet-activating factor acetylhydrolase. J. Lipid Res. 30:305-315.

56. Parthasarathy, S., and J. Barnett. 1990. Phospholipase $A_{2}$ activity of low density lipoprotein: evidence for an intrinsic phospholipase $A_{2}$ activity of apoprotein B-100. Proc. Natl. Acad. Sci. USA. 87:9741-9745. 
57. Cronstein, B. N., S. B. Kramer, G. Weissmann, and R. Hirschhorn. 1983. Adenosine: A physiological modulator of superoxide anion generation by human neutrophils. J. Exp. Med. 158:1160-1177.

58. Mayo, L. A., and J. T. Curnutte. 1990. Kinetic microplate assay for superoxide production by neutrophils and other phagocytic cells. Methods Enzymol. 186:567-575.

59. Ambrosio, G., J. L. Zweier, C. Duilio, P. Kuppusamy, G. Santoro, P. P. Elia, I. Tritto, P. Cirillo, M. Condorelli, M. Chiariello, and J. T. Flaherty. 1993. Evidence that mitochondrial respiration is a source of potentially toxic oxygen free radicals in intact rabbit hearts subjected to ischemia and reflow. J. Biol. Chem. 268:18532-18541.

60. Roius, M., F. Nigon, and J. Chapman. 1988. Platelet activating factor is a potent stimulant of the production of active oxygen species by human monocytederived macrophages. Biochem. Biophys. Res. Commun. 156:1293-1301.

61. Henderson, W. R., and M. Kaliner. 1978. Immunologic and nonimmunologic generation of superoxide from mast cells and basophils. J. Clin. Invest. 61:187-196.

62. Zweier, J. L., P. Kuppusamy, R. Williams, B. K. Rayburn, D. Smith, M. L. Weisfeldt, and J. T. Flaherty. 1989. Measurement and characterization of post ischemic free radical generation in the isolated perfused heart. J. Biol. Chem. 264:18890-18895.

63. Ambrosio, G., J. L. Zweier, and J. T. Flaherty. 1991. The relationship between oxygen radical generation and impairment of myocardial energy metabolism following postischemic reperfusion. J. Mol. Cell. Cardiol. 23:1359-1374.

64. Ambrosio, G., J. L. Zweier, W. E. Jacobus, M. L. Weisfeldt, and J. T. Flaherty. 1987. Improvement of post-ischemic myocardial function by administration of deferoxamine at the time of reflow. The role of iron in the pathogenesis of reperfusion injury. Circulation. 76:906-915.

65. Stephens, C. J., R. M. Graham, O. P. Yadava, L. L. Leong, M. J. Sturm, and R. R. Taylor. 1992. Plasma platelet activating factor degradation and serum lipids after coronary bypass surgery. Cardiovasc. Res. 26:25-31.

66. Royston, D., J. S. Fleming, J. B. Desai, S. Westaby, and K. M. Taylor 1986. Increased production of peroxidation products associated with cardiac operations. Evidence for free radical generation. J. Thorac. Cardiovasc. Surg. 91:759-766.

67. Prasad, K., J. Kalra, B. Bharadwaj, and A. K. Chaudhary. 1992. Increased oxygen free radical activity in patients on cardiopulmonary bypass undergoing aortocoronary bypass surgery. Am. Heart J. 123:37-45.

68. Kubes, P., M. Suzuki, and D. N. Granger. 1990. Modulation of PAF-induced leukocyte adherence and increased microvascular permeability. Am. $J$. Physiol. 259:G859-G864.

69. Cueva, J. P., and H. W. Hsue. 1988. Role of oxygen-derived free radicals in platelet-activating factor-induced bowel necrosis. Gut. 29:1207-1212.

70. Montrucchio, G., G. Alloatti, C. Tetta, R. De Luca, R. N. Saunders, G. Emmanueli, and G. Camussi. 1989. Release of platelet-activating factor (PAF) from ischemic-reperfused rabbit heart. Am. J. Physiol. 256:H1236-H1246.

71. Engler, R. L., M. D. Dahlgren, M. A. Peterson, A. Dobbs, and G. W. Schmid-Schonbein. 1986. Accumulation of polymorphonuclear leukocytes during 3-h experimental ischemia. Am. J. Physiol. 251:H93-H100.

72. Triggiani, M., and F. H. Chilton. 1992. Metabolism of platelet-activating factor in the guinea pig heart. J. Mol. Cell. Cardiol. 24:1101-1111.

73. McCord, J. M. 1974. Free radicals and inflammation: protection of synovial fluid by superoxide dismutase. Science (Wash. DC). 185:529-531.

74. Kuehl, F. A., J. L. Humes, R. W. Egan, E. A. Ham, G. C. Beveridge, and C. G. Van Arman. 1977. Role of prostaglandin endoperoxide $\mathrm{PGG}_{2}$ in inflammatory processes. Nature (Lond.). 265:170-173.

75. Shappel, S. B., A. A. Taylor, H. Hughes, J. R. Mitchell, D. C. Anderson, and C. W. Smith. 1990. Comparison of antioxidant and nonantioxidant lipoxygenase inhibitors on neutrophil function. Implications for pathogenesis of myocardial reperfusion injury. J. Pharmacol. Exp. Ther. 252:531-538.

76. Hallywell, B., J. R. Hoult, and D. R. Blake. 1988. Oxidants, inflammation, and anti-inflammatory drugs. FASEB (Fed. Am. Soc. Exp. Biol.) J. 2:28672873.

77. White, G. J. 1981. Inhibition of oxidative enzymes by antiallergy drugs. Agents Actions. 11:503-509. 